Moroccan J. of Pure and Appl. Anal. (MJPAA)

Volume 7(1), 2021, Pages 20-29

ISSN: Online 2351-8227 - Print 2605-6364

DOI: $10.2478 / \mathrm{mjpaa}-2021-0003$

\title{
Existence of positive solutions for second-order nonlinear neutral dynamic equations on time scales
}

\author{
Faycal Bouchelaghem $^{1}$, Abdelouaheb Ardjouni ${ }^{2}$, And Ahcene Djoudi ${ }^{3}$
}

\begin{abstract}
AвSTRACT. In this article we study the existence of positive solutions for second-order nonlinear neutral dynamic equations on time scales. The main tool employed here is Schauder's fixed point theorem. The results obtained here extend the work of Culakova, Hanustiakova and Olach [12]. Two examples are also given to illustrate this work.
\end{abstract}

Mathematics Subject Classification (2020). 34K13, 34A34, 34K30, 34L30.

Key words and phrases. Positive solutions, Schauder's fixed point theorem, Dynamic equations, Time scales.

Received : 04 June 2020 - Accepted: 17 October 2020.

(C)The Author(s) 2020. This article is published with open access by Sidi Mohamed Ben Abdallah University.

${ }^{1}$ High School of Management Sciences Annaba, Annaba 23000, Algeria,

Laboratory of Analysis and Control of Differential Equations "ACED", Fac. MISM, Dept. Maths, Univ 8 May 1945 Guelma, Guelma 24000, Algeria.

e-mail:fyb1500@gmail.com

2 Department of Mathematics and Informatics, Univ Souk Ahras, P.O. Box 1553, Souk Ahras 41000, Algeria, Applied Mathematics Lab., Faculty of Sciences, Department of Mathematics, Univ Annaba, P.O. Box 12, Annaba 23000, Algeria

e-mail: abd_ardjouni@yahoo.fr (Corresponding Author).

${ }^{3}$ Applied Mathematics Lab., Faculty of Sciences, Department of Mathematics, Univ Annaba, P.O. Box 12, Annaba 23000, Algeria

e-mail:adjoudi@yahoo.com. 


\section{Introduction}

Time scales calculus was initiated in 1988 by Stefan Hilger. It bridges the gap between continuous and discrete analysis and expands on both theories. Differential equations are defined on an interval of the set of real numbers while difference equations are defined on discrete sets. However, some physical systems are modeled by what is called dynamic equations because they are either differential equations, difference equations or a combination of both. This means that dynamic equations are defined on connected, discrete or combination of both types of sets. Hence, time scales calculus provides a generalization of differential and difference analysis, see $[6,7,14,15]$ and the references therein.

Delay dynamic equations arise from a variety of applications including in various fields of science and engineering such as applied sciences, practical problems concerning mechanics, the engineering technique fields, economy, control systems, physics, chemistry, biology, medicine, atomic energy, information theory, harmonic oscillator, nonlinear oscillations, conservative systems, stability and instability of geodesic on Riemannian manifolds, dynamics in Hamiltonian systems, etc. In particular, problems concerning qualitative analysis of delay dynamic equations have received the attention of many authors, see [1]-[15] and the references therein.

Let $\mathbb{T}$ be a time scale such that $t_{0} \in \mathbb{T}$. In this paper, we are interested in the analysis of qualitative theory of the problems of the existence of positive solutions for second-order nonlinear neutral dynamic equations. Inspired and motivated by the references in this paper, we concentrate on the existence of positive solutions for the second-order nonlinear neutral dynamic equation

$$
\left[r(t)\left(x(t)-P(t) x\left(t-\tau_{1}\right)\right)^{\Delta}\right]^{\Delta}+Q(t) f\left(x\left(t-\tau_{2}\right)\right)=0, t \geq t_{0}
$$

Throughout this paper we assume that $\tau_{1}>0, \tau_{2}>0, t-\tau_{1} \in \mathbb{T}$ and $t-\tau_{2} \in \mathbb{T}$, for all $t \geq t_{0}$, $r, P \in C_{r d}\left(\left[t_{0}, \infty\right) \cap \mathbb{T},(0, \infty)\right), Q \in C_{r d}(\mathbb{T},(0, \infty)), f$ is continuous nondecreasing function and $x f(x)>0, x \neq 0$.

By a solution of (1.1) we mean a function $x:\left[t_{1}-\tau_{1}, \infty\right) \cap \mathbb{T} \rightarrow \mathbb{R}$ is rd-continuous, for some $t_{1} \geq t_{0}$, such that both $x(t)-P(t) x\left(t-\tau_{1}\right)$ and $r(t)\left(x(t)-P(t) x\left(t-\tau_{1}\right)\right)^{\Delta}$ are rdcontinuously differentiable on $\left[t_{1}, \infty\right) \cap \mathbb{T}$ and such that (1.1) is satisfied for $t \geq t_{1}$.

To show the existence of positive solutions of (1.1), we transform (1.1) into an equivalent integral equation and then use Schauder's fixed point theorem. In the special case $\mathbb{T}=\mathbb{R}$, Culakova, Hanustiakova and Olach in [12] show the existence of positive solutions of (1.1) by using Schauder's fixed point theorem. Then, the results obtained here extend the work of Culakova, Hanustiakova and Olach [12].

One of papers related to our current topic is our work [8] where we investigated the existence and exponential stability of positive periodic solutions for a dynamic equation different from equation (1.1). More precisely, we studied the following second-order dynamic equation

$$
\left\{\begin{array}{l}
x^{\Delta \Delta}(t)+a\left(x^{\sigma}\right)^{\Delta}(t)+q(t) x^{\beta}(t)-r(t) x^{\alpha}(t)=0, t \geq t_{0} \\
x^{\Delta}\left(t_{0}\right)+a x^{\sigma}\left(t_{0}\right)=0, x\left(t_{0}\right)=1
\end{array}\right.
$$


The organization of this paper is as follows. In Section 2, we introduce some notations and definitions, and state some preliminary material needed in later sections. We will state some facts about the exponential function on a time scale as well as Schauder's fixed point theorem. For details on Schauder theorem we refer the reader to [16]. In Section 3, we are interested in the existence of a positive solution of (1.1) which is bounded with exponential functions by applying Schauder's fixed point theorem. The case when the solution converges to zero is also treated. In Section 4, we present two examples to illustrate our results.

\section{Preliminaries}

A time scale is an arbitrary nonempty closed subset of real numbers. The theory of dynamic equations unifies the theories of differential equations and difference equations. We suppose that the reader is familiar with the basic concepts concerning the calculus on time scales for dynamic equations. Otherwise one can find in the books $[6,7,15]$ most of the material needed to read this paper. We start by giving some definitions necessary for our work.

Definition 2.1 ([6]). We say that the function $f: \mathbb{T} \rightarrow \mathbb{R}$ is regulated if its right-sided limits exist at all right-dense points in $\mathbb{T}$ and its left-sided limits exist at all left-dense points in $\mathbb{T}$.

Definition 2.2 ([6]). A function $f: \mathbb{T} \rightarrow \mathbb{R}$ is called $r d$-continuous provided it is continuous at every right-dense point $t \in \mathbb{T}$ and its left-sided limits exist, and is finite at every left-dense point $t \in \mathbb{T}$. The set of $r$-continuous functions $f: \mathbb{T} \rightarrow \mathbb{R}$ will be denoted by

$$
C_{r d}=C_{r d}(\mathbb{T})=C_{r d}(\mathbb{T}, \mathbb{R}) \text {. }
$$

The set of functions $f: \mathbb{T} \rightarrow \mathbb{R}$ that are differentiable and whose derivative is $r d$-continuous is denoted by

$$
C_{r d}^{1}=C_{r d}^{1}(\mathbb{T})=C_{r d}^{1}(\mathbb{T}, \mathbb{R}) .
$$

Definition 2.3 ([6]). For $f: \mathbb{T} \rightarrow \mathbb{R}$, we define $f^{\Delta}(t)$ to be the number (if it exists) with the property that for any given $\varepsilon>0$, there exists a neighborhood $U$ of $t$ such that

$$
\left|(f(\sigma(t))-f(s))-f^{\Delta}(t)(\sigma(t)-s)\right|<\varepsilon|\sigma(t)-s| \text { for all } s \in U,
$$

where the forward jump operator $\sigma: \mathbb{T} \rightarrow \mathbb{T}$ is defined by

$$
\sigma(t)=\inf \{s \in \mathbb{T}: s>t\} \text { for all } t \in \mathbb{T} .
$$

The function $f^{\Delta}: \mathbb{T}^{k} \rightarrow \mathbb{R}$ is called the delta (or Hilger) derivative of $f$ on $\mathbb{T}^{k}=\mathbb{T} \backslash\{\sup \mathbb{T}\}$.

Definition 2.4 ([6]). A function $p: \mathbb{T} \rightarrow \mathbb{R}$ is called regressive provided

$$
1+\mu(t) p(t) \neq 0 \text { for all } t \in \mathbb{T}^{k},
$$

where the graininess function $\mu: \mathbb{T} \rightarrow[0, \infty)$ is defined by

$$
\mu(t)=\sigma(t)-t \text { for all } t \in \mathbb{T} \text {. }
$$

The set of all regressive and $r d$-continuous functions $p: \mathbb{T} \rightarrow \mathbb{R}$ will be denoted by $\mathcal{R}=\mathcal{R}(\mathbb{T}, \mathbb{R})$. We define the set $\mathcal{R}^{+}$of all positively regressive elements of $\mathcal{R}$ by

$$
\mathcal{R}^{+}=\mathcal{R}^{+}(\mathbb{T}, \mathbb{R})=\{p \in \mathcal{R}: 1+\mu(t) p(t)>0, \forall t \in \mathbb{T}\} .
$$


Definition 2.5 ([6]). If $p, q \in \mathcal{R}$, then the function $p \oplus q$ defined by

$$
(p \oplus q)(t):=p(t)+q(t)+\mu(t) p(t) q(t), \text { for all } t \in \mathbb{T} .
$$

and the function $\ominus p$ defined by

$$
(\ominus p)(t):=-\frac{p(t)}{1+\mu(t) p(t)} .
$$

Definition 2.6. We define the "circle minus" subtraction $\ominus$ on $\mathcal{R}$ by

$$
(p \ominus q)(t):=(p \oplus(\ominus q))(t) \text { for all } t \in \mathbb{T} .
$$

Lemma 2.1 ([6]). Suppose that $p, q \in \mathcal{R}$. Show directly from the definition that

(i) $p \ominus p=0$,

(ii) $\ominus(\ominus p)=p$,

(iii) $p \ominus q \in \mathcal{R}$,

(iv) $p \ominus q=\frac{p-q}{1+\mu q}$

(v) $\ominus(p \ominus q)=q \ominus p$,

(vi) $\ominus(p \oplus q)=(\ominus p) \oplus(\ominus q)$.

Theorem 2.1 ([6]). Suppose that $f: \mathbb{T} \rightarrow \mathbb{R}$ is a regulated function. Then there exists a function $F$ which is pre-differentiable with region of differentiation $D$ such that

$$
F^{\Delta}(t)=f(t) \text { for all } t \in D .
$$

Definition 2.7 ([6]). Suppose that $f: \mathbb{T} \rightarrow \mathbb{R}$ is a regulated function. We say that the function $F$ as in Theorem 2.1 is a pre-antiderivative of $f$. The indefinite integral of a regulated function $f$ is defined by

$$
\int f(t) \Delta t=F(t)+C,
$$

where $F$ is a pre-antiderivative of $f$ and $C$ is an arbitrary constant. The Cauchy integral is defined by

$$
\int_{s}^{t} f(t) \Delta t=F(t)-F(s) \text { for all } t, s \in \mathbb{T} .
$$

We say that a function $F: \mathbb{T} \rightarrow \mathbb{R}$ is an antiderivative of $f: \mathbb{T} \rightarrow \mathbb{R}$ if

$$
F^{\Delta}(t)=f(t) \text { for all } t \in \mathbb{T}^{k} .
$$

Theorem 2.2 ([6]). Every rd-continuous function has an antiderivative.

Definition 2.8 ([6]). Let $p \in \mathcal{R}$, then the generalized exponential function $e_{p}$ is defined as the unique solution of the initial value problem

$$
x^{\Delta}(t)=p(t) x(t), x(s)=1 \text {, where } s \in \mathbb{T} .
$$

An explicit formula for $e_{p}(t, s)$ is given by

$$
e_{p}(t, s)=\exp \left(\int_{s}^{t} \xi_{\mu(v)}(p(v)) \Delta v\right), \text { for all } s, t \in \mathbb{T},
$$


with

$$
\xi_{h}(v)=\left\{\begin{array}{l}
\frac{\log (1+h v)}{h} \text { if } h \neq 0, \\
v \quad \text { if } h=0,
\end{array}\right.
$$

where $\log$ is the principal logarithm function.

Lemma 2.2 ([6]). Let $p, q \in \mathcal{R}$. Then

(i) $e_{0}(t, s)=1$ and $e_{p}(t, t)=1$,

(ii) $e_{p}(\sigma(t), s)=(1+\mu(t) p(t)) e_{p}(t, s)$,

(iii) $e_{p}(t, s)=\frac{1}{e_{p}(s, t)}=e_{\ominus p}(s, t)$,

(iv) $e_{p}(t, s) e_{p}(s, r)=e_{p}(t, r)$

(v) $e_{p}(t, s) e_{q}(t, s)=e_{p \oplus q}(t, s)$,

(vi) $\left(\frac{1}{e_{p}(\cdot, s)}\right)^{\Delta}=-\frac{p(t)}{e_{p}^{\sigma}(\cdot, s)}$.

Lemma 2.3 ([1]). If $p \in \mathcal{R}^{+}$, then

$$
0<e_{p}(t, s) \leq \exp \left(\int_{s}^{t} p(v) \Delta v\right), \forall t \in \mathbb{T} .
$$

The proof of the main results in the next section is based upon an application of the following Schauder's fixed point theorem.

Theorem 2.3 (Schauder's fixed point theorem [16]). Let $\Omega$ be a closed, convex and nonempty subset of a Banach space $X$. Let $S: \Omega \rightarrow \Omega$ be a continuous mapping such that $S \Omega$ is a relatively compact subset of $X$. Then $S$ has at least one fixed point in $\Omega$. That is there exists an $x \in \Omega$ such that $S x=x$.

\section{Existence of positive solutions}

In this section we will study the existence of positive solutions of (1.1).

Theorem 3.1. Suppose that $0<k_{1}<k_{2}$,

$$
\int_{t_{0}}^{\infty} \xi_{\mu(v)}\left(k_{1} Q(v)\right) \Delta v=\infty
$$

and there exist $\gamma \geq 0$ such that $t_{0}-\gamma \in \mathbb{T}$,

$$
\frac{\ominus\left(k_{1} Q(t)\right)}{\ominus\left(k_{2} Q(t)\right)} e_{\left(k_{2} Q\right) \ominus\left(k_{1} Q\right)}\left(t_{0}, t_{0}-\gamma\right) \geq 1,
$$

and

$$
\begin{aligned}
& e_{\ominus\left(k_{2} Q\right)}\left(t, t-\tau_{1}\right)+e_{\left(k_{2} Q\right)}\left(t-\tau_{1}, t_{0}-\gamma\right) \int_{t}^{\infty} \frac{1}{r(s)} \int_{s}^{\infty} Q(\xi) \\
& \times f\left(e_{\ominus\left(k_{1} Q\right)}\left(\xi-\tau_{2}, t_{0}-\gamma\right)\right) \Delta \xi \Delta s \leq P(t) \leq e_{\ominus\left(k_{1} Q\right)}\left(t, t-\tau_{1}\right) \\
& +e_{\left(k_{1} Q\right)}\left(t-\tau_{1}, t_{0}-\gamma\right) \int_{t}^{\infty} \frac{1}{r(s)} \int_{s}^{\infty} Q(\xi) f\left(e_{\ominus\left(k_{2} Q\right)}\left(\xi-\tau_{2}, t_{0}-\gamma\right)\right) \Delta \xi \Delta s, t \geq t_{0} .
\end{aligned}
$$

Then (1.1) has a positive solution which tends to zero. 
Proof. We choose $T \geq t_{0}+\max \left\{\tau_{1}, \tau_{2}\right\}$ and set

$$
u(t)=e_{\ominus\left(k_{2} Q\right)}\left(t, t_{0}-\gamma\right), v(t)=e_{\ominus\left(k_{1} Q\right)}\left(t, t_{0}-\gamma\right), t \geq t_{0} .
$$

Let $X=C_{r d}\left(\left[t_{0}, \infty\right) \cap \mathbb{T}, \mathbb{R}\right)$ be the Banach space with the norm $\|x\|=\sup _{t \geq t_{0}}|x(t)|$. We now define a closed, bounded and convex subset $\Omega$ of $X$ as follows

$$
\Omega=\left\{x \in X: u(t) \leq x(t) \leq v(t), t \geq t_{0}\right\} .
$$

Define the operator $S: \Omega \longrightarrow X$ as follows

$$
(S x)(t)=\left\{\begin{array}{l}
P(t) x\left(t-\tau_{1}\right)-\int_{t}^{\infty} \frac{1}{r(s)} \int_{s}^{\infty} Q(\xi) f\left(x\left(\xi-\tau_{2}\right)\right) \Delta \xi \Delta s, t \geq T, \\
(S x)(T)+v(t)-v(T),
\end{array}\right.
$$

We will show that for any $x \in \Omega$ we have $S x \in \Omega$. For every $x \in \Omega$ and $t \geq T$ we get

$$
\begin{aligned}
(S x)(t) & \leq P(t) v\left(t-\tau_{1}\right)-\int_{t}^{\infty} \frac{1}{r(s)} \int_{s}^{\infty} Q(\xi) f\left(u\left(\xi-\tau_{2}\right)\right) \Delta \xi \Delta s \\
& =P(t) e_{\ominus\left(k_{1} Q\right)}\left(t-\tau_{1}, t_{0}-\gamma\right)-\int_{t}^{\infty} \frac{1}{r(s)} \int_{s}^{\infty} Q(\xi) f\left(e_{\ominus\left(k_{2} Q\right)}\left(\xi-\tau_{2}, t_{0}-\gamma\right)\right) \Delta \xi \Delta s \\
& \leq v(t) .
\end{aligned}
$$

For $t \in\left[t_{0}, T\right] \cap \mathbb{T}$ we have

$$
(S x)(t)=(S x)(T)+v(t)-v(T) \leq v(t) .
$$

Furthermore for $t \geq T$ we have

$$
\begin{aligned}
(S x)(t) & \geq P(t) u\left(t-\tau_{1}\right)-\int_{t}^{\infty} \frac{1}{r(s)} \int_{s}^{\infty} Q(\xi) f\left(v\left(\xi-\tau_{2}\right)\right) \Delta \xi \Delta s \\
& =P(t) e_{\ominus\left(k_{2} Q\right)}\left(t-\tau_{1}, t_{0}-\gamma\right)-\int_{t}^{\infty} \frac{1}{r(s)} \int_{s}^{\infty} Q(\xi) f\left(e_{\ominus\left(k_{1} Q\right)}\left(\xi-\tau_{2}, t_{0}-\gamma\right)\right) \Delta \xi \Delta s \\
& \geq u(t) .
\end{aligned}
$$

Finally let $t \in\left[t_{0}, T\right] \cap \mathbb{T}$ and set

$$
H(t)=v(t)-v(T)-u(t)+u(T) .
$$

Then with regard to (3.2) it follows that

$$
\begin{aligned}
H^{\Delta}(t) & =v^{\Delta}(t)-u^{\Delta}(t)=\ominus\left(k_{1} Q(t)\right) v(t)-\left(\ominus k_{2} Q(t)\right) u(t) \\
& =v(t)\left[\ominus\left(k_{1} Q(t)\right)-\ominus\left(k_{2} Q(t)\right) u(t) e_{k_{1} Q}\left(t, t_{0}-\gamma\right)\right] \\
& =v(t)\left[\ominus\left(k_{1} Q(t)\right)-\ominus\left(k_{2} Q(t)\right) e_{\ominus\left(k_{2} Q\right) \oplus\left(k_{1} Q\right)}\left(t, t_{0}-\gamma\right)\right] \\
& =v(t)\left[\ominus\left(k_{1} Q(t)\right)-\ominus\left(k_{2} Q(t)\right) e_{\left(\ominus\left(k_{2} Q\right)\right) \oplus\left(k_{1} Q\right)}\left(t_{0}, t_{0}-\gamma\right)\right] \leq 0,
\end{aligned}
$$

Since $H(T)=0$ and $H^{\Delta}(t) \leq 0$ for $t \in\left[t_{0}, T\right] \cap \mathbb{T}$, this leads to the conclusion that

$$
H(t)=v(t)-v(T)-u(t)+u(T) \geq 0, t \in\left[t_{0}, T\right] \cap \mathbb{T} .
$$

Then for $t \in\left[t_{0}, T\right] \cap \mathbb{T}$ and any $x \in \Omega$ we get

$$
(S x)(t)=(S x)(T)+v(t)-v(T) \geq u(T)+v(t)-v(T) \geq u(t) .
$$


Thus we have proved that $S x \in \Omega$ for any $x \in \Omega$.

We now show that $S$ is continuous. Let $x_{i} \in \Omega$ be such that $x_{i} \longrightarrow x$ as $i \longrightarrow \infty$. Because $\Omega$ is closed, $x \in \Omega$. For $t \geq T$, we have

$$
\begin{aligned}
& \left|\left(S x_{i}\right)(t)-(S x)(t)\right| \\
& \leq P(t)\left|x_{i}\left(t-\tau_{1}\right)-x\left(t-\tau_{1}\right)\right| \\
& +\left|\int_{t}^{\infty} \frac{1}{r(s)} \int_{s}^{\infty} Q(\xi)\left[f\left(x_{i}\left(\xi-\tau_{2}\right)\right)-f\left(x\left(\xi-\tau_{2}\right)\right)\right] \Delta \xi \Delta s\right| \\
& \leq P(t)\left|x_{i}\left(t-\tau_{1}\right)-x\left(t-\tau_{1}\right)\right|+\int_{t}^{\infty} \frac{1}{r(s)} \int_{s}^{\infty} Q(\xi)\left|f\left(x_{i}\left(\xi-\tau_{2}\right)\right)-f\left(x\left(\xi-\tau_{2}\right)\right)\right| \Delta \xi \Delta s .
\end{aligned}
$$

Since $\left|f\left(x_{i}\left(\xi-\tau_{2}\right)\right)-f\left(x\left(\xi-\tau_{2}\right)\right)\right| \rightarrow 0$ as $i \rightarrow \infty$, by applying the Lebesgue dominated convergence theorem we obtain that

$$
\lim _{i \longrightarrow \infty}\left\|S x_{i}-S x\right\|=0 .
$$

This means that $S$ is continuous.

We now show that $S \Omega$ is relatively compact. It is sufficient to show by the Arzela-Ascoli theorem that the family of function $\{S x: x \in \Omega\}$ is uniformly bounded and equicontinuous on $\left[t_{0}, \infty\right) \cap \mathbb{T}$. The uniform boundedness follows from the definition of $\Omega$. For the equicontinuity we only need to show that for any given $\varepsilon>0$ the interval $\left[t_{0}, \infty\right) \cap \mathbb{T}$ can be decomposed into finite subintervals in such a way that on each subinterval all functions of the family have change of amplitude less than $\varepsilon$. Then for any $\varepsilon>0$ we take $T^{*} \geq T$ large enough so that $(S x)\left(T^{*}\right) \leq \frac{\varepsilon}{2}$.

For $x \in \Omega, T^{*} \leq T_{1}<T_{2}$ we get

$$
\left|(S x)\left(T_{2}\right)-(S x)\left(T_{1}\right)\right| \leq\left|(S x)\left(T_{2}\right)\right|+\left|(S x)\left(T_{1}\right)\right| \leq \frac{\varepsilon}{2}+\frac{\varepsilon}{2}=\varepsilon .
$$

For $x \in \Omega, T \leq T_{1}<T_{2} \leq T^{*}$ we have

$$
\begin{aligned}
& \left|(S x)\left(T_{2}\right)-(S x)\left(T_{1}\right)\right| \\
& \leq\left|P\left(T_{2}\right) x\left(T_{2}-\tau_{1}\right)-P\left(T_{1}\right) x\left(T_{1}-\tau_{1}\right)\right|+\int_{T_{1}}^{T_{2}} \frac{1}{r(s)} \int_{s}^{\infty} Q(\xi) f\left(x\left(\xi-\tau_{2}\right)\right) \Delta \xi \Delta s \\
& \leq\left|P\left(T_{2}\right) x\left(T_{2}-\tau_{1}\right)-P\left(T_{1}\right) x\left(T_{1}-\tau_{1}\right)\right| \\
& +\left(T_{2}-T_{1}\right) \max _{T_{1} \leq s \leq T_{2}}\left\{\frac{1}{r(s)} \int_{s}^{\infty} Q(\xi) f\left(x\left(\xi-\tau_{2}\right)\right) \Delta \xi \Delta s\right\} .
\end{aligned}
$$

Thus there exists a $\delta>0$ such that

$$
\left|(S x)\left(T_{2}\right)-(S x)\left(T_{1}\right)\right| \leq \varepsilon \text { if } 0<T_{2}-T_{1}<\delta .
$$

Finally for any $x \in \Omega, t_{0} \leq T_{1}<T_{2} \leq T$ there exists a $\delta>0$ such that

$$
\left|(S x)\left(T_{2}\right)-(S x)\left(T_{1}\right)\right|=\left|v\left(T_{2}\right)-v\left(T_{1}\right)\right| \leq \varepsilon \text { if } 0<T_{2}-T_{1}<\delta .
$$

Then $\{S x: x \in \Omega\}$ is uniformly bounded and equicontinuous on $\left[t_{0}, \infty\right) \cap \mathbb{T}$ and hence $S \Omega$ is a relatively compact subset of $C_{r d}\left(\left[t_{0}, \infty\right) \cap \mathbb{T}, \mathbb{R}\right)$. By Lemma 2.3 there is an $x_{0} \in \Omega$ such that $S x_{0}=x_{0}$. We see that $x_{0}$ is a positive solution of (1.1). The proof is complete. 
Corollary 3.1. Suppose that $k>0$, (3.1) holds and

$$
P(t)=e_{\ominus(k Q)}\left(t, t-\tau_{1}\right)+e_{(k Q)}\left(t-\tau_{1}, t_{0}\right) \int_{t}^{\infty} \frac{1}{r(s)} \int_{s}^{\infty} Q(\xi) f\left(e_{\ominus(k Q)}\left(\xi-\tau_{2}, t_{0}\right)\right) \Delta \xi \Delta s, t \geq t_{0} .
$$

Then (1.1) has a solution

$$
x(t)=e_{\ominus(k Q)}\left(t, t_{0}\right), t \geq t_{0} .
$$

Proof. We put $k_{1}=k_{2}=k, \gamma=0$ and apply Theorem 3.1.

\section{Two examples}

In this section, we give two examples to illustrate the applications of Theorem 3.1 and corollary 3.1.

Example 4.1. Consider the nonlinear neutral dynamic equation on $\mathbb{T}=\mathbb{Z}$,

$$
\left[r(t)\left(x(t)-P(t) x\left(t-\tau_{1}\right)\right)^{\Delta}\right]^{\Delta}+q f\left(x\left(t-\tau_{2}\right)\right)=0, t \geq t_{0} .
$$

where $Q(t)=q=2>0, \tau_{1}=2, \tau_{2}=1, f(t)=t, r(t)=2^{t}, t_{0}=1$. For $k_{1}=1, k_{2}=2, \gamma=1$ we have

and

$$
\begin{gathered}
e_{\left(k_{2} Q\right)}\left(t_{0}, t_{0}-\gamma\right)=\exp \left(\int_{t_{0}-\gamma}^{t_{0}} \log \left(1+k_{2} q\right) \Delta v\right)=\left(1+k_{2} q\right)^{\gamma}, \\
e_{\left(k_{2} Q\right) \ominus\left(k_{1} Q\right)}\left(t_{0}, t_{0}-\gamma\right)=\left(\frac{1+k_{2} q}{1+k_{1} q}\right)^{\gamma},
\end{gathered}
$$

$$
\frac{\ominus\left(k_{1} Q(t)\right)}{\ominus\left(k_{2} Q(t)\right)} e_{\left(k_{2} Q\right) \ominus\left(k_{1} Q\right)}\left(t_{0}, t_{0}-\gamma\right)=\frac{-\frac{k_{1} q}{1+k_{1} q}}{-\frac{k_{2} q}{1+k_{2} q}}\left(\frac{1+k_{2} q}{1+k_{1} q}\right)^{\gamma}=\frac{1}{2}\left(\frac{5}{3}\right)^{2}=\frac{25}{18} \text {. }
$$

Then

$$
\begin{gathered}
\frac{\ominus\left(k_{1} Q(t)\right)}{\ominus\left(k_{2} Q(t)\right)} e_{\left(k_{2} Q\right) \ominus\left(k_{1} Q\right)}\left(t_{0}, t_{0}-\gamma\right) \geq 1, \\
e_{\ominus\left(k_{2} Q\right)}\left(t, t-\tau_{1}\right)+e_{\left(k_{2} Q\right)}\left(t-\tau_{1}, t_{0}-\gamma\right) \int_{t}^{\infty} \frac{1}{r(s)} \int_{s}^{\infty} Q(\xi) f\left(e_{\ominus\left(k_{1} Q\right)}\left(\xi-\tau_{2}, t_{0}-\gamma\right)\right) \Delta \xi \Delta s \\
=\left(1+k_{2} q\right)^{-\tau_{1}}+\left(1+k_{2} q\right)^{-\tau_{1}-t_{0}+\gamma}\left(1+k_{1} q\right)^{t_{0}-\gamma+\tau_{2}+2} \frac{2}{1+2 k_{1} q}\left(\frac{1+k_{2} q}{2+2 k_{1} q}\right)^{t} \\
=\frac{1}{25}+\frac{2}{3375}\left(\frac{5}{6}\right)^{t},
\end{gathered}
$$

and

$$
\begin{aligned}
& e_{\ominus\left(k_{1} Q\right)}\left(t, t-\tau_{1}\right)+e_{\left(k_{1} Q\right)}\left(t-\tau_{1}, t_{0}-\gamma\right) \int_{t}^{\infty} \frac{1}{r(s)} \int_{s}^{\infty} Q(\xi) f\left(e_{\ominus\left(k_{2} Q\right)}\left(\xi-\tau_{2}, t_{0}-\gamma\right)\right) \Delta \xi \Delta s \\
& =\left(1+k_{1} q\right)^{-\tau_{1}}+\left(1+k_{1} q\right)^{-\tau_{1}-t_{0}+\gamma}\left(1+k_{2} q\right)^{t_{0}-\gamma+\tau_{2}+2} \frac{2}{1+2 k_{2} q}\left(\frac{1+k_{1} q}{2+2 k_{2} q}\right)^{t}
\end{aligned}
$$




$$
=\frac{1}{9}+\frac{2}{10125}\left(\frac{3}{10}\right)^{t} \text {. }
$$

Therefore

$$
\frac{1}{25}+\frac{2}{3375}\left(\frac{5}{6}\right)^{t} \leq P(t) \leq \frac{1}{9}+\frac{2}{10125}\left(\frac{3}{10}\right)^{t}, t \geq 1
$$

If $P(t)$ fulfils the inequalities above, then all conditions of Theorem 3.1 are satisfied and (4.1) has a positive solution which is bounded by functions $u(t)=e_{\ominus\left(k_{2} Q\right)}\left(t, t_{0}-\gamma\right)=(5)^{-t}, v(t)=e_{\ominus\left(k_{1} Q\right)}\left(t, t_{0}-\right.$ $\gamma)=(3)^{-t}$ and tends to zero.

Example 4.2. Consider the nonlinear neutral dynamic equation on $\mathbb{T}=h \mathbb{Z}, h>0$,

$$
\left[2^{t}\left(x(t)-P(t) x\left(t-\tau_{1}\right)\right)^{\Delta}\right]^{\Delta}+q x\left(t-\tau_{2}\right)=0, t \geq t_{0} .
$$

where $q, \tau_{1}, \tau_{2} \in(0, \infty)$ and

$$
\begin{aligned}
P(t) & =e_{\ominus(k Q)}\left(t, t-\tau_{1}\right)+e_{(k Q)}\left(t-\tau_{1}, t_{0}\right) \int_{t}^{\infty} \frac{1}{r(s)} \int_{s}^{\infty} q e_{\ominus(k Q)}\left(\xi-\tau_{2}, t_{0}\right) \Delta \xi \Delta s \\
& =(1+k h q)^{-\frac{\tau_{1}}{h}}+(1+k h q)^{\frac{-\tau_{1}+\tau_{2}+2}{h}} q \frac{2 h^{2}}{(1+k h q)^{\frac{1}{h}}-12(1+k h q)^{\frac{1}{h}}-1} \frac{1}{2\left(\frac{1}{2}\right)^{t}, t \geq t_{0}}
\end{aligned}
$$

where $k \in(0, \infty)$. Since all conditions of Corollary 3.1 are satisfied, then (4.2) has a positive solution

$$
x(t)=(1+k h q)^{\frac{t_{0}-t}{h}}, t \geq t_{0} .
$$

which tends to zero.

Acknowledgements. The authors would like to thank the anonymous referee for his/her valuable comments and good advice.

Conflict of interest. The authors declare that they have no conflict of interest.

\section{References}

[1] M. Adivar and Y. N. Raffoul, Existence of periodic solutions in totally nonlinear delay dynamic equations, Electronic Journal of Qualitative Theory of Differential Equations 2009(1) (2009), 1-20.

[2] A. Ardjouni, A. Djoudi, Existence of positive periodic solutions for nonlinear neutral dynamic equations with variable delay on a time scale, Malaya Journal of Matematik 2(1) (2013), 60-67.

[3] A. Ardjouni, A. Djoudi, Existence of periodic solutions for nonlinear neutral dynamic equations with functional delay on a time scale, Acta Univ. Palacki. Olomnc., Fac. rer. nat., Mathematica 52(1) (2013), 5-19.

[4] A. Ardjouni, A. Djoudi, Existence of periodic solutions for nonlinear neutral dynamic equations with variable delay on a time scale, Commun Nonlinear Sci Numer Simulat 17 (2012), 3061-3069.

[5] A. Ardjouni, A. Djoudi, Periodic solutions in totally nonlinear dynamic equations with functional delay on a time scale, Rend. Sem. Mat. Univ. Politec. Torino Vol. 68, 4(2010), 349-359.

[6] M. Bohner, A. Peterson, Dynamic Equations on Time Scales, An Introduction with Applications, Birkhäuser, Boston, 2001.

[7] M. Bohner, A. Peterson, Advances in Dynamic Equations on Time Scales, Birkhäuser, Boston, 2003.

[8] F. Bouchelaghem, A. Ardjouni, A. Djoudi, Existence and exponential stability of positive periodic solutions for second-order dynamic equations, Ural Math. J. 6(1) (2020), 42-53. 
[9] F. Bouchelaghem, A. Ardjouni, A. Djoudi, Existence and stability of positive periodic solutions for delay nonlinear dynamic equations, Nonlinear Studies 25(1) (2018), 191-202.

[10] F. Bouchelaghem, A. Ardjouni, A. Djoudi, Existence of positive solutions of delay dynamic equations, Positivity 21(4) (2017), 1483-1493.

[11] F. Bouchelaghem, A. Ardjouni, A. Djoudi, Existence of positive periodic solutions for delay dynamic equations, Proyecciones (Antofagasta) 36(3) (2017), 449-460.

[12] I. Culakova, L. Hanustiakova, R. Olach, Existence for positive solutions of second-ordre neutral nonlinear differential equations, Applied Mathematics Letters 22 (2009), 1007-1010.

[13] M. Gouasmia, A. Ardjouni, A. Djoudi, Periodic and nonnegative periodic solutions of nonlinear neutral dynamic equations on a time scale, International Journal of Analysis and Applications 16(2) (2018), 162-177.

[14] S. Hilger, Ein Masskettenkalkül mit Anwendung auf Zentrumsmanningfaltigkeiten. PhD thesis, Universität Würzburg, 1988.

[15] V. Lakshmikantham, S. Sivasundaram, B. Kaymarkcalan, Dynamic Systems on Measure Chains, Kluwer Academic Publishers, Dordrecht, 1996.

[16] D. R. Smart, Fixed Points Theorems, Cambridge Univ. Press, Cambridge, UK, 1980. 